\title{
Genetische Analyse der Population des Vorderwälder Rindes
}

\begin{abstract}
Title of the paper: Genetic analysis of the population of Vorderwald cattle

The actual population of Vorderwald cattle, comprising 5452 animals has been analysed concerning the gene contribution, the generation interval and the degree of relationship and inbreeding with regard of five generations of ancestors.

About $84 \%$ of the genes are originating from Vorderwald cattle. The genetic portions of other breeds have been contributed by Red Holsteins, Montbéliards and Ayrshires. The average generation interval parents-current animals is about 4.5 years. The mean relationship has been found out with $5.5 \%$ within the bulls and $3.4 \%$ within the cows. Accordingly the mean coefficient of inbreeding is higher within the bulls $(2,7 \%)$ than within the cows (2,4 \%). Only $28.6 \%$ of the bulls and $36.6 \%$ of the cows are not inbred. With $0.9 \%$ the increase of inbreeding in the last generation is of alarming proportions; that's why a carefully directed program of mating has to be recommended.
\end{abstract}

Key Words: Vorderwald, gene contribution, generation interval, degree of relationship, coefficient of inbreeding, rate of inbreeding

\section{Zusammenfassung}

Die aktuelle, 5452 Tiere umfassende Zuchtpopulation des Vorderwälder Rindes wurde unter Einbeziehung von fünf Vorfahrengenerationen hinsichtlich ihrer Fremdgenanteile, des Generationsintervalls sowie der Verwandtschafts- und Inzuchtverhältnisse untersucht.

Etwa 84 \% der Genanteile sind Vorderwälder Ursprungs. Fremdgenanteile stammen vorrangig von den Rassen Red-Holstein, Montbéliard und Ayrshire. Das durchschnittliche Generationsintervall Eltern-Probanden beträgt ca. 4,5 Jahre. Die mittlere Verwandtschaft wurde innerhalb der Bullen mit 5,5 \%, innerhalb der Kühe mit 3,4 \% festgestellt. Dementsprechend wurde für die Bullen ein etwas höherer durchschnittlicher Inzuchtkoeffizient von 2,7 \% als für die Kühe mit 2,4 \% geschätzt. Nur 28,6 \% der Bullen und 36,6 \% der Kühe sind nicht ingezogen. Die Inzuchtrate in der letzten Generation der Gesamtpopulation besitzt mit $0,9 \%$ ein bedenkliches Ausmaß, so dass für die Zukunft ein gezieltes Paarungsprogramm zu empfehlen ist.

Schlüsselwörter: Vorderwälder, Genanteile, Generationsintervall, Verwandtschaft, Inzuchtkoeffizient, Inzuchtrate

\section{1. $\quad$ Einleitung}

Die Wahrung genetischer Diversität hat sich in jüngerer Zeit zu einem allgemeinen Anliegen in der Tierzucht entwickelt. Der Verlust an genetischen Ressourcen bezieht sich sowohl auf die Verarmung hinsichtlich Rassenvielfalt als auch auf die Minderung genetischer Variabilität innerhalb der Populationen und ist das Ergebnis der Selektion zwischen bzw. innerhalb von Populationen (GROENEVELD, 2003). Von der Verringerung genetischer Vielfalt sind vor allem kleine, vielfach in ihrer Existenz bedrohte Populationen betroffen. Gleichzeitig stellt sich in ihnen vermehrt die Inzucht als zusätzliches Problem ein.

Während einige Rassen, wie etwa das Hinterwälder Rind, unter dem Aspekt ihrer Erhaltung besondere Aufmerksamkeit auf sich ziehen, scheinen andere weniger Beach- 
tung zu finden; letzteres trifft für das, wie die Hinterwälder im Schwarzwald beheimatete Vorderwälder Rind zu. Allerdings umfasst seine Herdbuchpopulation noch immer ca. 5.400 Tiere, so dass es in der Roten Liste für Rinderrassen lediglich in die Kategorie „zur Bestandsbeobachtung“ eingruppiert wurde; eine akute Gefährdung scheint somit derzeit noch nicht zu bestehen.

Die Erhaltungsperspektiven des Wälderviehs sind eng mit der Situation der landwirtschaftlichen Betriebe im Schwarzwald verbunden. Neben der Pflege der dortigen Kulturlandschaft verleiht es seinem Verbreitungsgebiet ein wesentliches Markenzeichen, womit es gleichzeitig einen Beitrag zum Tourismus leistet, dem im Schwarzwald hohe wirtschaftliche Bedeutung zukommt. Die ungünstigen Standortbedingungen (Höhenlage, verkürzte Vegetationszeit, Topographie) und strukturellen Nachteile haben ökonomische Erschwernisse zur Folge, so dass, trotz aller staatlichen Fördermaßnahmen, die Milchviehhaltung einen permanenten Schrumpfungsprozess erlebte. Der Anteil der „gesicherten Betriebe“ (Betriebe mit Hofnachfolger oder jungem Betriebsleiter) im Schwarzwald wird durch das baden-württembergische Ministerium für Ernährung und Ländlichen Raum mit lediglich 59 \% veranschlagt, so dass besagte Entwicklung auch in Zukunft anzuhalten droht. Schließlich wurden die Vorderwälder in flacheren Vorbergzonen, aber auch auf den Hochflächen, vielfach durch andere milchleistungsstärkere Rassen ersetzt.

Neben dem Inzuchtproblem leiden kleine Populationen infolge geringeren Zuchtfortschrittes unter dem wachsenden Leistungsrückstand gegenüber großen Populationen. Um diesen Problemen zu begegnen, bediente man sich im Laufe der Zuchtgeschichte des Vorderwälder Rindes wiederholt der Einkreuzung fremder Rassen (Fleckvieh, Ayrshire, Red Holstein, Montbéliard).

Dies gab Veranlassung, die derzeitige genetische Zusammensetzung der Vorderwälder Rasse sowie die Inzucht- sowie Verwandtschaftsverhältnisse der Population zu untersuchen.

\section{$2 . \quad$ Material und Methoden}

Die Zuchtbuchauszüge aller im Frühjahr 2003 aktiven Vorderwälder Herdbuchtiere lieferten die Datengrundlage für die Analyse. Die Bullendaten umfassten neben den im Natursprung verwendeten Vatertieren auch jene, deren Sperma sich zum Erfassungstermin im Einsatz befand. Somit konnten 5452 Zuchttiere, davon 5263 Kühe und 189 Bullen einschließlich, soweit bekannt, ihrer Vorfahren in die Untersuchung einbezogen werden. Die Zahl der Herdbuchkühe entspricht ungefähr einem Anteil von 29 \% am gesamten Vorderwälder Kuhbestand (BRODAUF, 2003).

Die Auswertungen wurden mit Hilfe des Programms OPTI-MATE Vers. 3.82 von WREDE und SCHMIDT (2003) durchgeführt. Mit Rücksicht auf die in älteren Vorfahrengenerationen der Pedigrees feststellbaren Lücken und im Interesse der Vergleichbarkeit mit früheren entsprechenden Untersuchungen basieren sie generell auf fünf Ahnengenerationen.

Anhand der Rassezugehörigkeit der Vorfahren der fünften Ahnengeneration konnten die Genanteile für jeden Probanden und daraus die genetische Zusammensetzung der Population geschätzt werden.

Die Generationsintervalle wurden als durchschnittliches Alter der jeweiligen Eltern bei der Geburt ihrer Nachkommen berechnet. Falls lediglich das Geburtsjahr bekannt war, 
wurde, um Fehler auf ein Minimum zu begrenzen, der 01.07. ersatzweise als Geburtstag eingesetzt.

Die von WRIGHT (1923) eingeführte Pedigree-Methode diente zur Schätzung von Inzucht- und Verwandtschaftskoeffizienten der Einzeltiere, deren Mittelung die entsprechenden durchschnittlichen Populationsparameter lieferte. WIGGANS et al. (1995), LUTAAYA et al. (1999), BAUMUNG und SÖLKNER (2002) sowie PIRCHNER (2002) weisen allerdings auf die Grenzen der Pedigree-Analyse hin, da unvollständige Stammbäume zwangsläufig Fehler bei der Schätzung von Inzuchtkoeffizienten nach sich ziehen. Um diese zu minimieren, wurde der von WREDE und SCHMIDT (2003) beschriebene Vollständigkeitsindex (VI) zu deren Korrektur verwendet.

Die Schätzung der Inzuchtrate $\Delta \mathrm{F}$ wurde unterschiedlich, wie folgt, vorgenommen:

$\Delta \mathrm{F}_{1} \quad$ Mittlere Inzuchtrate pro Generation seit der 5. Ahnengeneration:

$$
\Delta \mathrm{F}_{1}=\frac{\mathrm{F}_{\mathrm{t}}}{\mathrm{n}-1} \quad \begin{array}{cl}
\mathrm{F}_{\mathrm{t}} & \begin{array}{l}
\text { durchschnittlicher Inzuchtkoeffizient der aktuellen } \\
\text { Population }
\end{array} \\
\mathrm{n} & =\text { Anzahl berücksichtigter Ahnengenerationen }
\end{array}
$$

$\Delta \mathrm{F}_{2} \quad$ Wie $\Delta \mathrm{F}_{1}$, aber unter Berücksichtigung der Pedigreevollständigkeit

$\Delta \mathrm{F}_{3} \quad$ Inzuchtrate in der letzten Generation:

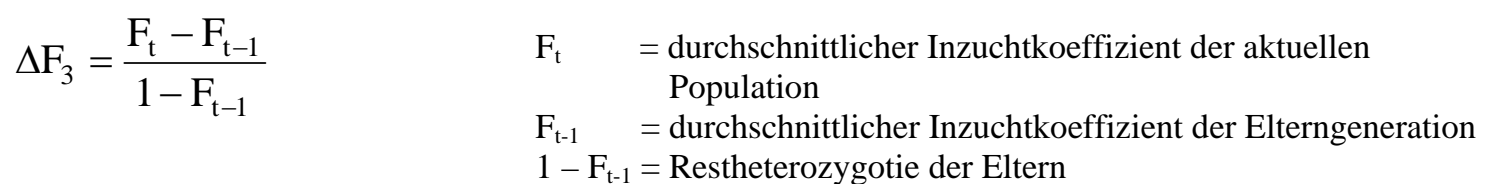

$\Delta \mathrm{F}_{4} \quad$ Wie $\Delta \mathrm{F}_{3}$, aber unter Berücksichtigung der Pedigreevollständigkeit

$\Delta \mathrm{F}_{5} \quad$ Erwartete Inzuchtrate aufgrund der effektiven Anzahl Väter und Mütter:

$$
\Delta \mathrm{F}_{5}=\frac{1}{8 \cdot \mathrm{M}_{\mathrm{e}}}+\frac{1}{8 \cdot \mathrm{W}_{\mathrm{e}}} \quad \begin{array}{ll}
\mathrm{M}_{\mathrm{e}} & =\text { effektive Anzahl männlicher Zuchttiere } \\
\mathrm{W}_{\mathrm{e}} & =\text { effektive Anzahl weiblicher Zuchttiere }
\end{array}
$$

3. Ergebnisse

3.1. Genanteile fremder Rassen

Insgesamt beträgt der Fremdgenanteil in der Vorderwälder Population 5,8 \%; bei den Bullen stellt er sich geringfügig höher (6,4 \%) heraus (Tab. 1). Daran sind vorerst die Red Holsteins noch am stärksten beteiligt, lediglich bei den Bullen macht sich der Montbéliard-Einfluss bereits deutlich bemerkbar. Der Genanteil unbekannter Herkunft nimmt mit ungefähr einem Drittel einen beträchtlichen Umfang ein. Dass er bei den Bullen etwas geringer ausfällt, dürfte mit der exakteren Zuchtbuchführung bei den Vatertieren im Zusammenhang stehen.

Tabelle 1

Genanteile der Herkunftsrassen (\%) innerhalb der aktuellen Population (Gene contribution of different breeds (per cent) within the current population)

\begin{tabular}{lcrr}
\hline Rassen & Gesamtpopulation & Bullen & Kühe \\
\hline Vorderwälder & 60,83 & 62,30 & 60,78 \\
Red-Holstein & 4,96 & 3,92 & 4,99 \\
Montbéliard & 0,56 & 2,41 & 0,50 \\
Ayrshire & 0,23 & 0,05 & 0,24 \\
Schwarzbunte & 0,06 & 0,02 & 0,06 \\
Sonstige Rassen* & 0,01 & $<0,01$ & 0,01 \\
unbekannt & 33,35 & 31,30 & 33,42 \\
\hline
\end{tabular}

*Braunvieh, Jersey, Hinterwälder, Fleckvieh, Limousin 
Der hohe Anteil unbekannter Herkunft lässt sich möglicherweise mit Eintragungen der 1970er Jahre erklären, die ohne Nachweis einer gesicherten Abstammung getätigt wurden. Ferner ist anzunehmen, dass der Genanteil der Red Holsteins infolge zum Teil unkontrollierten Spermaeinsatzes unterschätzt ist und es sich bei Tieren mit unbekannter Abstammung vielfach um Abkömmlinge der Red Holstein-Rasse handelt. Seitens der Zuchtleitung wird dementsprechend deren Genanteil mit 15 \% wesentlich höher eingeschätzt (BRODAUF, 2003). Unterstellt man die Richtigkeit dieser Annahme und dass die Genanteile innerhalb der unbekannten Herkünfte ansonsten jener der bekannten Herkünfte entsprechen, so resultiert die in Tabelle 2 angegebene Verteilung der Herkunftsrassen, womit der Anteil Vorderwälder Blutes auf 82-84 \% anwächst. Möglicherweise verbindet sich damit eine bessere Annäherung an die reale genetische Zusammensetzung der Population.

Tabelle 2

Genanteile der Herkunftsrassen (\%) innerhalb der aktuellen Population unter Vernachlässigung der unbekannten Ahnen (Gene contribution of different breeds (per cent) within the current population neglecting unknown ancestors)

\begin{tabular}{lcrc}
\hline Rassen & Gesamtpopulation & Bullen & Kühe \\
\hline Vorderwälder & 83,82 & 81,74 & 83,89 \\
Red Holstein & 15,00 & 15,00 & 15,00 \\
Montbéliard & 0,77 & 3,16 & 0,69 \\
Ayrshire & 0,32 & 0,07 & 0,33 \\
Schwarzbunte & 0,08 & 0,03 & 0,08 \\
sonstige Rassen* & 0,01 & $<0,01$ & 0,01 \\
\hline * Braunvieh, Jersey, Hinterwälder, Fleckvieh, Limousin & &
\end{tabular}

* Braunvieh, Jersey, Hinterwälder, Fleckvieh, Limousin

\subsection{Generationsintervalle}

Innerhalb der gesamten sowie der Kuh-Population unterscheiden sich die Generationsintervalle Väter-Probanden und Mütter-Probanden nur geringfügig (Tab. 3). Anders verhält es sich bei den Bullen, deren Väter im Durchschnitt bei ihrer Geburt um ca. 1,8 Jahre älter sind als deren Mütter und zudem das mittlere Alter der Kuhväter um ca. 2,4 Jahre übertreffen.

Tabelle 3

Mittlere Generationsintervalle (Jahre) (Mean generation intervals (years))

\begin{tabular}{lccc}
\hline Pfad & Gesamtpopulation & Bullen & Kühe \\
\hline Eltern - Probanden & 4,46 & 5,78 & 4,40 \\
& $(1,96-12,44)$ & $(1,98-10,27)$ & $(1,98-12,44)$ \\
Väter - Probanden & 4,41 & 6,69 & 4,31 \\
& $(1,73-12,03)$ & $(1,73-9,77)$ & $(1,78-12,03)$ \\
Mütter - Probanden & 4,51 & 4,87 & 4,50 \\
& $(2,18-12,84)$ & $(2,23-10,76)$ & $(2,18-12,84)$ \\
\hline
\end{tabular}

Ergänzend wird in der Abbildung die Entwicklung der Generationsintervalle innerhalb der Bullenväter und -mütter sowie der Kuhväter und -mütter dargestellt. Sowohl bei den Bullen- als auch Kuhvätern erhöhten sich die Generationsintervalle, bei letzteren allerdings weniger deutlich und auf geringerem Niveau. Hingegen zeigt sich bei den Bullen- und Kuhmüttern im Zuge der Generationen eine erhebliche Abnahme des Durchschnittsalters bei Geburt ihrer Nachkommen. 


\subsection{Inzucht}

3.3.1.1. Verwandtschaft innerhalb der Population

Verbunden mit deutlich höherer Vollständigkeit ihrer Stammbäume existiert bei den Bullen im Durchschnitt eine engere Verwandtschaft als bei den weiblichen Zuchttieren (Tab. 4), wenngleich innerhalb letzterer der höchste maximale Einzelverwandtschaftskoeffizient gefunden wurde. Dem entspricht die Beobachtung, dass nur ca. 1,6 \% der Bullenpaare in keiner verwandtschaftlichen Beziehung stehen (Kühe über $14 \%$ ) und bei mehr als 94 \% der Fälle (Kühe ca. 85 \%) die Verwandtschaft bis zu 20 \% beträgt.

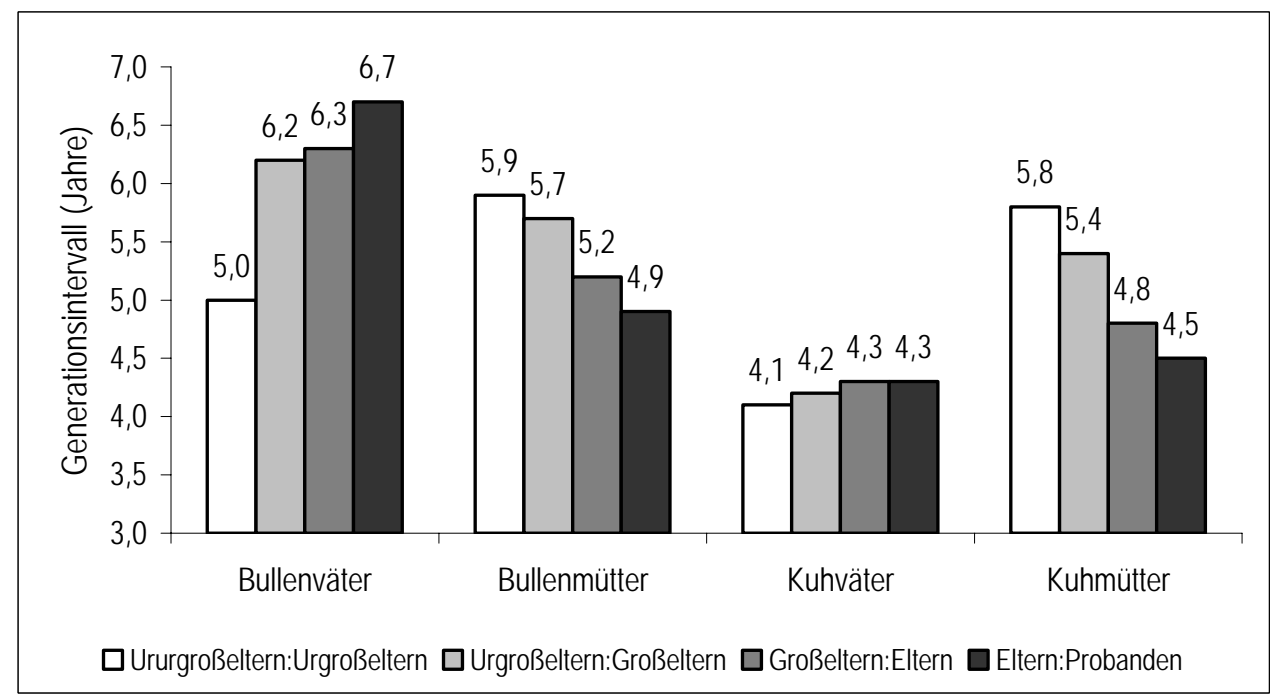

Abb.: Entwicklung der Generationsintervalle der Bullen- und Kuhväter und -mütter (Development of the generation intervals of the male and female ancestors of bulls and cows)

Die mittlere verwandtschaftliche Beziehung zwischen Bullen und Kühen ist mit 3,7 \% zwischen jener innerhalb der Bullen und innerhalb der Kühe angesiedelt. Nur knapp 9 \% aller Tierpaare sind frei von verwandtschaftlicher Bindung, während über $90 \%$ aller Bullen-Kuh-Paare von einem Verwandtschaftskoeffizienten bis zu 20 \% gekennzeichnet sind.

Tabelle 4

Verwandtschaftskoeffizienten (oben) und deren Verteilung (unten; in \%) (Degrees of relationship (above) and the distribution of them (below; per cent))

\begin{tabular}{lcccc}
\hline Verwandtschaft & Gesamtpop. & Bullen & Kühe & Bullen:Kühe \\
\hline $\begin{array}{l}\text { Mittlerer Verwandtschaftskoeff. } \overline{\mathrm{R}} \\
\text { (\%) }\end{array}$ & 3,44 & 5,48 & 3,41 & 3,73 \\
Max. Verwandtschaftskoeff. $\mathrm{R}_{\max }(\%)$ & 65,63 & 62,89 & 65,63 & 62,50 \\
Mittlerer Vollständigkeitsindex & 59,55 & 67,83 & 59,26 & 63,40 \\
$\overline{\mathrm{VI}}(\%)$ & & & & \\
\hline \multicolumn{1}{c}{$\mathrm{R}=0$} & 14,22 & 1,57 & 14,63 & 8,75 \\
& 80,52 & 85,09 & 80,18 & 85,17 \\
$10<\mathrm{R} \leq 10$ & 4,44 & 9,23 & 4,38 & 5,11 \\
$20<\mathrm{R} \leq 30$ & 0,73 & 3,35 & 0,72 & 0,84 \\
$30<\mathrm{R} \leq 40$ & 0,08 & 0,69 & 0,08 & 0,11 \\
$40<\mathrm{R}$ & 0,01 & 0,08 & 0,01 & 0,01 \\
\hline
\end{tabular}

\subsection{Inzuchtkoeffizienten}

Wie in Tabelle 5 ersichtlich, ist der mittlere Inzuchtkoeffizient $\left(\overline{\mathrm{F}}_{1}\right)$ bei den Bullen von höherem Ausmaß als jener der Kühe bzw. der Gesamtpopulation, wenngleich für diese 
ein bedeutend höherer maximaler Einzelwert von mehr als $25 \%$ festgestellt wurde. Dementsprechend erweisen sich weniger Stiere (28,6 \%) als nicht ingezogen als dies für die Kühe zutrifft (36,6 \%). Zudem sind die Pedigrees der Bullen von höherer Vollständigkeit gekennzeichnet. Werden die Vollständigkeitsindices in die Schätzung der Inzuchtkoeffizienten einbezogen, so fallen diese $\left(\bar{F}_{2}\right)$ wesentlich höher aus, wobei die Relation zwischen Bullen und Kühen weitgehend erhalten bleibt.

\section{Tabelle 5}

Inzuchtkoeffizienten (oben) und deren Verteilung (unten; in \%) (Coefficients of inbreeding (above) and the contribution of them (below; in per cent))

\begin{tabular}{|c|c|c|c|}
\hline Inzuchtkoeffizient & Gesamtpopulation & Bullen & Kühe \\
\hline Mittlerer Inzuchtkoeffizient $\overline{\mathrm{F}}_{1}(\%)$ & 1,42 & 1,75 & 1,41 \\
\hline Maximaler Inzuchtkoeffizient $\mathrm{F}_{1 . \max }(\%)$ & 25,20 & 12,50 & 25,20 \\
\hline Mittlerer Vollständigkeitsindex $\overline{\mathrm{VI}}(\%)$ & 59,62 & 63,92 & 59,47 \\
\hline Mittlerer Inzuchtkoeffizient $\overline{\mathrm{F}}_{2}(\%)$ & 2,38 & 2,74 & 2,37 \\
\hline$F_{1}=0,000$ & 36,35 & 28,57 & 36,63 \\
\hline $0,001 \leq \mathrm{F}_{1} \leq 1,000$ & 22,32 & 26,46 & 22,17 \\
\hline $1,001 \leq \mathrm{F}_{1} \leq 2,000$ & 17,79 & 16,40 & 17,84 \\
\hline $2,001 \leq \mathrm{F}_{1} \leq 3,000$ & 6,69 & 5,82 & 6,71 \\
\hline $3,001 \leq \mathrm{F}_{1} \leq 4,000$ & 8,51 & 10,05 & 8,46 \\
\hline $4,001 \leq \mathrm{F}_{1} \leq 5,000$ & 2,82 & 4,23 & 2,77 \\
\hline $5,001 \leq \mathrm{F}_{1} \leq 6,000$ & 1,14 & 1,59 & 1,12 \\
\hline $6,001 \leq \mathrm{F}_{1} \leq 7,000$ & 1,85 & 4,23 & 1,77 \\
\hline $7,001 \leq \mathrm{F}_{1} \leq 8,000$ & 1,10 & 1,59 & 1,08 \\
\hline $8,001 \leq \mathrm{F}_{1} \leq 9,000$ & 0,20 & 0,00 & 0,21 \\
\hline $9,001 \leq \mathrm{F}_{1} \leq 10,000$ & 0,33 & 0,00 & 0,34 \\
\hline $10,001 \leq \mathrm{F}_{1}$ & 0,89 & 1,06 & 0,83 \\
\hline
\end{tabular}

\subsection{Inzuchtrate}

Im Durchschnitt der zurückliegenden fünf Generationen betrug die Inzuchtzunahme je Generation $\left(\Delta \mathrm{F}_{1}\right)$ bei den Bullen etwas mehr als bei den Kühen (Tab. 6). An dieser Feststellung ändert sich nichts nach Korrektur mittels der Vollständigkeitsindices $\left(\Delta \mathrm{F}_{2}\right)$, davon abgesehen, dass die mittleren Inzuchtraten höhere Werte annehmen. Dabei erweisen sich die Inzuchtzuwächse von der Elterngeneration zur Generation der aktuellen Tiere in allen Fällen höher als die entsprechenden Durchschnittswerte von fünf Generationen. Dies gilt sowohl für die unkorrigierten $\left(\Delta \mathrm{F}_{3}\right)$ als auch korrigierten Inzuchtraten $\left(\Delta \mathrm{F}_{4}\right)$. Im Falle letzterer fällt besonders der relativ hohe Wert $1,14 \%$ bei den Bullen auf. Auf der Grundlage der effektiven Anzahl von Vater- und Muttertieren ergibt sich eine künftig zu erwartende Inzuchtrate $\left(\Delta \mathrm{F}_{5}\right)$ innerhalb der Kühe und der Gesamtpopulation von 0,10\%, innerhalb der Bullen von 0,46 \%.

Tabelle 6

Inzuchtrate (Rate of inbreeding)

\begin{tabular}{cccc}
\hline Inzuchtrate & Gesamtpopulation & Bullen & Kühe \\
\hline$\Delta \mathrm{F}_{1}$ & 0,36 & 0,44 & 0,35 \\
$\Delta \mathrm{F}_{2}$ & 0,60 & 0,68 & 0,59 \\
$\Delta \mathrm{F}_{3}$ & 0,56 & 0,72 & 0,55 \\
$\Delta \mathrm{F}_{4}$ & 0,86 & 1,14 & 0,85 \\
$\Delta \mathrm{F}_{5}$ & 0,10 & 0,46 & 0,10 \\
\hline
\end{tabular}




\subsection{Bedeutende Ahnen und Inzuchtverursacher}

Anhand der durchschnittlichen Verwandtschaft der Vorfahren zur aktuellen Population lassen sich die bedeutendsten Ahnen ermitteln. In Tabelle 7 werden die fünf wichtigsten Vorfahren genannt. Neben deren mittlerer Verwandtschaft zu den Tieren der aktuellen Population, werden die durchschnittliche Generation sowie die Häufigkeit ihres Auftretens als Elter angegeben. Zudem enthält die Tabelle die fünf bedeutendsten Inzuchtverursacher mit ihrer Anzahl der zur Inzucht der aktuellen Tiere führenden Bindungen sowie ihrem Beitrag zu deren durchschnittlichen Inzucht. Entsprechend ihrer geringen Nachkommenzahl sind in beiden Listen, von einer Ausnahme (Simone) abgesehen, keine weiblichen Tiere anzutreffen.

Insgesamt tritt die Red Holstein-blütige M-Linie mit dem Bullen Max (100\% RH), dessen Sohn Markig (50 \% RH) und Enkel Marc (25 \% RH) stark hervor. Der Bulle Marschall entstammt der Paarung von Marc und der Kuh Simone (50 \% RH). Unter den bedeutendsten Ahnen der Bullen machen sich mit Gardian und Espada bereits zwei Vertreter der Montbéliard-Rasse bemerkbar. In der Reihe der Inzuchtverursacher sind zusätzlich die beiden Halbbrüder Top (50 \% RH) und Toster (50\% RH, 6,25 \% Braunvieh) zu nennen. Der Bulle Feldsee schließlich trägt neben 9,38 \% Ayrshire- und 0,79 \% Schwarzbunt-Blut einen Red-Holstein-Genanteil von 18,75 \%.

Tabelle 7

Bedeutendste Ahnen und Inzuchtverursacher (The most important ancestors and those with highest contribution to the inbreeding of the probands)

\begin{tabular}{|c|c|c|c|c|c|c|c|c|}
\hline \multicolumn{5}{|c|}{ Bedeutendste Ahnen } & \multicolumn{4}{|c|}{ Bedeutendste Inzuchtverursacher } \\
\hline Name & $\begin{array}{l}\text { Ge- } \\
\text { burt }\end{array}$ & $\begin{array}{c}\text { durchschn. } \\
\text { direkte } \\
\text { Verwandtsch. } \\
(\%)\end{array}$ & $\begin{array}{c}\text { durchschn. } \\
\text { Generation } \\
\text { des Auftretens }\end{array}$ & $\begin{array}{l}\text { Häufig- } \\
\text { keit des } \\
\text { Auftre- } \\
\text { tens }\end{array}$ & Name & $\begin{array}{l}\text { Ge- } \\
\text { burt }\end{array}$ & $\begin{array}{c}\text { Anzahl } \\
\text { Bindungen }\end{array}$ & $\begin{array}{l}\text { Beitrag zur } \\
\text { durchschn. } \\
\text { Inzucht (\%) }\end{array}$ \\
\hline & \multicolumn{8}{|c|}{ Gesamtpopulation } \\
\hline Markig & 1978 & 10,41 & 4,25 & 8829 & Markig & 1978 & 929 & 28,71 \\
\hline Marschall & 1982 & 8,88 & 3,32 & 3829 & Marschall & 1982 & 464 & 24,23 \\
\hline Marc & 1980 & 6,91 & 4,03 & 5154 & Marc & 1980 & 325 & 9,27 \\
\hline Max & 1975 & 5,03 & 4,59 & 5918 & Feldsee & 1986 & 71 & 4,43 \\
\hline \multirow[t]{2}{*}{ Simone } & 1979 & 4,31 & 4,09 & 3374 & Toster & 1979 & 68 & 2,58 \\
\hline & \multicolumn{8}{|c|}{ Bullen } \\
\hline Markig & 1978 & 12,37 & 4,34 & 365 & Marschall & 1982 & 24 & 34,60 \\
\hline Marschall & 1982 & 9,44 & 3,51 & 159 & Markig & 1978 & 27 & 21,80 \\
\hline Gardian & 1991 & 9,13 & 1,40 & 43 & Feldsee & 1986 & 10 & 7,27 \\
\hline Marc & 1980 & 6,37 & 4,19 & 192 & Top & 1978 & 1 & 5,54 \\
\hline \multirow[t]{2}{*}{ Espada } & 1989 & 5,82 & 1,98 & 42 & Marc & 1980 & 7 & 3,46 \\
\hline & \multicolumn{8}{|c|}{ Kühe } \\
\hline Markig & 1978 & 10,34 & 4,24 & 8464 & Markig & 1978 & 902 & 29,01 \\
\hline Marschall & 1982 & 8,86 & 3,31 & 3670 & Marschall & 1982 & 440 & 23,78 \\
\hline Marc & 1980 & 6,93 & 4,02 & 4962 & Marc & 1980 & 318 & 9,52 \\
\hline Max & 1975 & 5,03 & 4,59 & 5732 & Feldsee & 1986 & 61 & 4,31 \\
\hline Simone & 1979 & 4,31 & 4,09 & 3246 & Toster & 1979 & 66 & 2,64 \\
\hline
\end{tabular}

\section{4.} Diskussion

\section{Fremdgenanteile}

Die Vorderwälder Rasse musste im Laufe ihrer Zuchtgeschichte des 20. Jahrhunderts mehrere Einkreuzungswellen über sich ergehen lassen. Die Einfuhr und intensive Einkreuzung von Simmentalern um 1900 und während der Folgejahre machte den Anfang und hatte eine ernsthafte Bedrohung der Rasse zur Folge. Mit dem Ziel, die an Bedeutung zunehmende Milchleistung und dem durch angrenzende Rassen wachsenden 
Konkurrenzdruck zu begegnen, entschloss man sich Ende der 1960er Jahre für den Einsatz von Ayrshire-Bullen (Egmont, Forecast, Sypland Officer, Uppermost). Gleichzeitig wurde damit die Verbesserung von Euterausbildung und Melkbarkeit angestrebt. Um die Inzuchtgefahr infolge starken Überhandnehmens einer Ayrshire-Linie zu verhindern und der anhaltenden Konkurrenz seitens anderer Rassen entgegenzutreten, bediente man sich Ende der 1970er Jahre des Einsatzes von fünf kanadischen Red Holstein-Bullen (Feuer, Max, Royal, Topper, Walter). Mitte der 1990er Jahre sahen sich die Vorderwälder Züchter erneut den Problemen früherer Jahre, nämlich unbefriedigende Entwicklung der Milchleistung und deutliche Linienverknappung, konfrontiert. Dies veranlasste zur vorerst letzten Einkreuzung von fünf französischen Montbéliard-Bullen (Bistro, Brac, Brachy, Espada, Gardian). Alle genannten Einkreuzungsversuche waren mit von den Fremdrassen herrührenden Nachteilen unterschiedlicher Art verbunden.

Weitere gelegentlich benutzte Vatertiere anderer Rassen (Braunvieh, Jersey, Hinterwälder, Limousin) machen sich bei der Schätzung von Fremdgenanteilen kaum bemerkbar. Zumindest bei Berücksichtigung von fünf Ahnengenerationen lassen sich auch Fleckviehanteile kaum mehr nachweisen. Die mit geringfügigen Genanteilen vertretenen Schwarzbunten haben offensichtlich ohne Konzept der Züchtervereinigung Eingang in die Vorderwälder Population gefunden. Der Ayrshire-Einfluss scheint innerhalb der aktuellen Population auf einen unbedeutenden Rest zurück gedrängt worden zu sein.

Der geschätzte durchschnittliche Red Holstein-Anteil dürfte nicht der Realität entsprechen; seitens der Zuchtverantwortlichen wird er mit ca. 15 \% wesentlich höher veranschlagt. Der Montbéliard-Einfluss ist vorerst vor allem bei den Bullen festzustellen; es kann erwartet werden, dass er sich in naher Zukunft nicht nur bei diesen, sondern auch in der Kuhpopulation in größerem Umfang ausweiten wird.

Nach Umlegung der unbekannten Herkünfte auf die bekannten entsprechend der für diese festgestellten Verteilung, kann der Vorderwälder Rasse ein wesentlich höheres $\mathrm{Ma}$ an Rassereinheit unterstellt werden, auch wenn sie diesbezüglich, zumindest in Teilen, der Hinterwälder Rasse unterlegen sein dürfte, für die BIEDERMANN et al. (2003) einen genetischen Reinheitsgrad von ca. 90 \% geschätzt haben.

Aus jüngerer Zeit liegen vergleichbare Untersuchungen an Deutschen Schwarzbunten alter Zuchtrichtung vor (EHLING et al., 1999), die rasseeigene Genanteile von mehr als 99 \% nachwiesen. Entsprechende Schätzungen für das württembergische Braunvieh und die westfälischen Rotbunten (BOLLMEIER et al., 1991; SCHMIDT et al., 1993), mit denen die der jeweiligen Rasse eigenen Genanteile mit 22 bis 77 \% festgestellt wurden, dürften die derzeitigen Verhältnisse allerdings nicht mehr repräsentieren.

\section{Generationsintervalle}

Im Interesse des Zuchtfortschrittes ist, entsprechend einer Studie von OLLIVIER (1974), bei Bullen eine möglichst kurze, bei Kühen hingegen eine möglichst lange Nutzungsdauer anzustreben. Im Falle ausschließlichen Natursprungs sollte das optimale Abgangsalter der Vatertiere sogar deutlich geringer ausfallen als bei weiblichen Zuchttieren. Gemessen an den Generationsintervallen in der Hinterwälder Population (BIEDERMANN et al., 2003) mit nur geringen Anteilen (15 \%) der künstlichen Besamung und Generationsintervallen Väter-Nachkommen von ca 3,5 Jahren bzw. Mütter-Nachkommen von 6,5 Jahren treffen derartige „ideale“ Relationen weitgehend zu. 
In der Vorderwälder Population macht sich der höhere Anteil der künstlichen Besamung von 44,5 \% zumindest hinsichtlich des mittleren Generationsintervalls Bullenväter-männliche Nachkommen mit 6,7 Jahren im Vergleich zu jenem zwischen Müttern und Nachkommen von 4,9 bzw. 4,5 Jahren bemerkbar. Auffallend ist allerdings das um ca. 2,4 Jahre höhere Durchschnittsalter der Väter bei Geburt ihrer männlichen Nachkommen gegenüber jenem bei Geburt ihrer weiblichen Nachkommen. Es ist daher anzunehmen, dass Bullenväter häufiger in der künstlichen Besamung anzutreffen sind als Kuhväter. Hinzu kommt, dass Anfang der 1990er Jahre, mit dem Ziel einer „Linienaktivierung“, Samen ehemaliger Bullenväter (z. B. Attila, Berserker, Burger, Fermit, Toster, Wald) erneut im Rahmen des Zuchtprogramms zum Einsatz gelangten. Dass im Zuge der zurückliegenden Generationen die Generationsintervalle innerhalb der Väter angestiegen sind - bei den Bullenvätern bedeutend stärker als bei den Kuhvätern - dürfte die Folge des in der gleichen Zeit gewachsenen Anteils der künstlichen Besamung sein.

Die eindeutige Dominanz der künstlichen Besamung erklärt auch die deutlich höheren Generationsintervalle für den Pfad Väter-Nachkommen im Vergleich zu jenen zwischen den Müttern und ihren Nachkommen in der deutschen Schwarz- und Rotbuntzucht (SCHMIDT, 1993; ENGELHARDT, 1996).

Vergleicht man schließlich die Generationsintervalle Mütter-Nachkommen (4,5-4,9 Jahre) mit entsprechenden Werten, die SCHMIDT et al. (1993) und ENGELHARDT (1996) mit 4,2 bzw. 4,6 Jahren fanden, so lässt sich diesbezüglich für das Vorderwälder Rind kein Vorteil ableiten. Gegenüber den Hinterwäldern und der Genreserve schwarzbunter Rinder (4,9-6,5 Jahre) (EHLING et al., 1999) macht sich sogar eine deutlich ungünstigere Situation bemerkbar. Dementsprechend beträgt das derzeitige Durchschnittsalter der Vorderwälder Herdbuch-Kühe mit 5,7 Jahren zwar mehr als das aller Herdbuchkühe in Deutschland (4,8 Jahre), aber erheblich weniger als dies für die Hinterwälder Kühe mit 7,0 Jahren zutrifft (ADR, 2003). Damit sind die Vorderwälder Kühe seit 1971 im Mittel um 1,8 Jahre jünger geworden (ADR, 1972). Diese bedauerliche Entwicklung spiegelt sich seit den zurückliegenden vier Generationen im kontinuierlichen Rückgang der Generationsintervalle Mütter-männliche Nachkommen und Mütter-weibliche Nachkommen wider.

\section{Verwandtschaft und bedeutende Ahnen}

Die für die Vorderwälder ermittelten durchschnittlichen Verwandtschaftsgrade innerhalb der Bullen und Kühe sowie zwischen Bullen und Kühen übertreffen die Schätzungen für das württembergische Braunvieh und die Hinterwälder (BOLLMEIER et al., 1991; BIEDERMANN et al., 2003). Lediglich für die schwarzbunte Genreserve Niedersachsens (EHLING et al., 1999) stellte sich zwar eine geringere Verwandtschaft innerhalb der Kühe, jedoch ein wesentlich höherer Verwandtschaftsgrad innerhalb der Bullen heraus. Für Kühe der britischen HF-Population schätzten ROUGHSEDGE et al. (1999) den Verwandtschaftsgrad mit nur 1,34 \%; überraschend mutet allerdings der außerordentlich hohe Wert an, den YOUNG und SEYKORA (1996) für die große Holstein-Population der USA mit 10,2 \% angeben.

Die relativ enge Verwandtschaft innerhalb der Vorderwälder Population lässt sich sicherlich auf den in den zurückliegenden Jahrzehnten überaus starken Einsatz von Bullen weniger Linien zurückführen. Tatsächlich finden sich unter den fünf bedeutendsten Ahnen der Gesamtpopulation und der Kühe vier Bullen der M-Linie, die durch den Red-Holstein-Bullen Max begründet wurde. Mit den Montbéliard-Bullen 
Espada und Gardian scheint sich, vorerst auf die Bullen beschränkt, ein Wechsel abzuzeichnen.

\section{Inzucht und bedeutende Inzuchtverursacher}

Wegen der Gefahr der Entstehung von Inzuchtdepressionen bzw. des möglicherweise vermehrten Auftretens von Erbdefekten muss vor allem in kleinen Populationen der Inzucht ständige Aufmerksamkeit gewidmet werden. Hohe Inzuchtraten bzw. eine geringe effektive Populationsgröße verbinden sich gleichzeitig mit einem schleichenden Verlust an genetischer Variabilität (SIMIANER und KÖNIG, 2003), der in der Zuchtplanung entsprechende Berücksichtigung finden sollte..

Die für die Vorderwälder Population auf der Basis von fünf Ahnengenerationen geschätzten Inzuchtkoeffizienten übertreffen in ihrer Höhe die meisten entsprechenden Schätzwerte, die bei in- und ausländischen Rinderpopulationen gewonnen wurden und innerhalb der Grenzen 0,3 und 1,7 \% angesiedelt sind (HAGGER, 1988: schweizerisches Braunvieh; MIGLIOR et al., 1990: kanadische Jerseys und Holsteins; BOLLMEIER et al., 1991: württembergisches Braunvieh; SCHMIDT et al., 1993: westfälische Rotbunte; NAVAJAS und URIOSTE, 1995: argentinische Aberdeen-Angus; YOUNG und SEYKORA, 1996: US-amerikanische Holsteins; KROGMEIER et al., 1997: süddeutsches Gelb- und Braunvieh; EHLING et al., 1999: schwarzbunte Genreserve Niedersachsens, ausgenommen Besamungsbullen; ROUGHSEDGE et al., 1999: britische Holstein-Friesians; BIEDERMANN et al., 2003: Hinterwälder). Es gilt allerdings zu bedenken, dass die Schätzungen durch vorgenannte Autoren teilweise der aktuellen Situation der betreffenden Populationen nicht mehr entsprechen dürften, bzw. zum Teil für wesentlich umfangreichere Populationen gelten. Schätzwerte der Inzucht aus neueren Untersuchungen von CASSEL et al. (2002 a, b) für amerikanische Holsteins und Jerseys im Bereich zwischen 2,4 und 5,1 \% geben den deutlichen Hinweis, dass Inzucht auch in großen Populationen zu einem Problem anwachsen kann.

Für die Situation in der Vorderwälder Population dürfte vorrangig der übermäßig starke Einsatz einiger Bullen bzw. Bullenlinien als Erklärung für die relativ hohen Inzuchtgrade in Frage kommen. Tatsächlich lieferten alleine die ein und derselben Linie angehörenden Bullen Markig, Marschall und Marc zur durchschnittlichen Inzucht der Kuhpopulation einen Beitrag von 62 \%, zu jener der Bullen von 60 \%.

Die effektive Populationsgröße $\mathrm{N}_{\mathrm{e}}$ betrachtet man im allgemeinen als den entscheidenden Maßstab, um die Gefährdungssituation von Populationen zu bewerten. Nach der Empfehlung des Ausschusses der DGfZ zur Erhaltung genetischer Vielfalt bei landwirtschaftlichen Nutztieren (1992) sollte im Interesse des langfristigen Erhalts einer Population $\mathrm{N}_{\mathrm{e}}$ nicht weniger als 50 betragen; dies entspräche einer Inzuchtrate von $\Delta \mathrm{F}=1 \%$. Damit verbindet sich allerdings, entsprechend der Vermutung von BREM et al. (1990) ein weitgehender Verzicht auf Selektion und Zuchtfortschritt. Um noch erfolgreich selektieren zu können, nehmen dieselben Autoren eine effektive Populationsgröße von mindestens $100(\Delta \mathrm{F}=0,5 \%)$ als untere Grenze an. GROENEVELD (2003) berichtet über das 2002 von oben genanntem DGfZ-Ausschuss verabschiedete „Nationale Fachprogramm zur Erhaltung und Nutzung tiergenetischer Ressourcen“, wonach eine Population mit einer effektiven Populationsgröße von weniger als 200 $(\Delta \mathrm{F}>0,25 \%)$ als stark existenzgefährdet zu betrachten ist, so dass unmittelbar Erhaltungsmaßnahmen einzuleiten sind. Es wird zudem empfohlen, eine Kryokonserve an- 
zulegen, sobald $\mathrm{N}_{\mathrm{e}}$ weniger als 1000 beträgt ( $\left.\Delta \mathrm{F}>0,05 \%\right)$. Lediglich bei $\mathrm{N}_{\mathrm{e}}>1000$ kann demnach eine Population als nicht bedroht eingestuft werden; sie sollte jedoch unter ständiger Beobachtung stehen.

Die für die Vorderwälder Population geschätzten Inzuchtraten $\left(\Delta \mathrm{F}_{1}\right.$ bis $\left.\Delta \mathrm{F}_{4}\right)$ bewegen sich somit, legt man die neueren durch die DGfZ angegebenen Grenzwerte zugrunde, im Bereich der Existenzgefährdung. Im Falle der Bullen übersteigt $\Delta \mathrm{F}_{4}$ sogar den Wert $1 \%$. Zudem hat die Inzuchtrate in den zurückliegenden Generationen eine Zunahme erfahren, denn die Inzuchtsteigerung in der letzten Generation zeigt sich von höherem Ausmaß als im Durchschnitt der letzten fünf Generationen. Die Population nähert sich somit einem recht bedenklichen Inzuchtniveau.

Gemessen an der Zahl der Zuchtkühe und Bullen wäre eine erheblich geringere Inzuchtzunahme realisierbar, wie die, allerdings gleich verteilten Bulleneinsatz voraussetzende $\mathrm{zu}$ erwartende Inzuchtrate $\Delta \mathrm{F}_{5}$ verdeutlicht. Im Vergleich zu Einnutzungsrassen verfügt die als Zweinutzungsrasse gezüchtete Vorderwälder Population ohnehin über günstigere Voraussetzungen, den Inzuchtzuwachs gering zu halten, wie PIRCHNER (2002) anhand der, wenn auch bedeutend größeren bayerischen Fleckvieh-Population trotz hoher $\mathrm{KB}$-Anteile $(\Delta \mathrm{F}=0,13-0,16 \%)$ sowie der sehr kleinen Population des Tiroler Grauviehs ( $\Delta \mathrm{F}=0,51 \%)$ aufzeigen konnte. Ähnlich gilt dies für die Hinterwälder; obwohl nach Abspaltung einer Mutterkuhpopulation die Milchviehpopulation nur ein Siebtel der Vorderwälder Rasse umfasst, erfuhr diese im Durchschnitt von fünf Generationen eine weitaus geringere Steigerung der Inzucht (BIEDERMANN et al., 2003).

Untersuchungen für andere Rinderpopulationen (BOLLMEIER et al, 1991: württembergisches Braunvieh; MIGLIOR und BURNSIDE, 1995: kanadische Holsteins; SCHMIDT et al., 1993: westfälische Rotbunte; YOUNG und SEYKORA, 1996: USamerikanische Holstein-Kühe; KROGMEIER et al., 1997: süddeutsches Gelb- und Braunvieh; EHLING et al., 1999: schwarzbunte Genreserve Niedersachsens; PIRCHNER, 2002: bayerisches Fleckvieh) zeitigten ebenfalls geringere Inzuchtraten, die innerhalb der Grenzen 0,13 und 0,44 variieren.

Das Problem sinkender genetischer Variabilität infolge eines zu starken Einsatzes von Vatertieren nur weniger Linien hat sich in der Vorderwälder Zucht wiederholt bemerkbar gemacht, so dass jeweils in der Einkreuzung fremder Rassen der Rettungsanker gesehen wurde. In der jüngeren Zeit handelte es sich um die Bevorzugung der MLinie, die schließlich zur jüngsten Einkreuzungswelle mit Montbéliard-Bullen veranlasste, womit allerdings gleichzeitig dem weiteren Verlust an Eigenständigkeit der Rasse Vorschub geleistet wird.

Es muss daher vermehrt darauf geachtet werden, dass, soweit als möglich, verwandte Tiere nicht zur Paarung gelangen, dass sich der Einsatz von Vererbern nicht auf Angehörige weniger Linien konzentriert, sondern die verfügbare Linienvielfalt vermehrt ausgeschöpft wird, bzw. versucht wird, für eine gleichmäßigere Verteilung der Vatertiere auf die Kühe zu sorgen. Letzteres ist den Züchtern sicherlich nicht problemlos zu vermitteln und verlangt von diesen im Interesse des Schicksals der Population und einer nachhaltigen Zucht, in der Zuchtfortschritt und Inzuchtzunahme in gesunder Balance stehen, ein hohes Maß an Einsehen. Keinesfalls darf auf die Anlage von Sicherheit bildenden Spermareserven unverwandter Bullen verzichtet werden. 


\section{Danksagung}

Der Rinder-Union Baden-Württemberg wird für die Bereitstellung der Zuchtbuchauszüge, Herrn J. Wrede, Institut für Tierzucht und Vererbungsforschung der Tierärztlichen Hochschule Hannover für die vielfältige Hilfestellung herzlich gedankt.

\section{Literatur}

ADR:

Rinderproduktion in der Bundesrepublik Deutschland 1971, 2002. (1972, 2003)

BAUMUNG, R.; SÖLKNER, J.:

Analysis of pedigrees of Tux-Zillertal, Carinthian Blond and Original Pinzgau cattle populations in Austria. J. Anim. Breed. Genet. 119 (2002), 175-181

BIEDERMANN, G.; WALDMANN, S.; MAUS, F.:

Genetische Analyse der Population des Hinterwälder Rindes. Arch. Tierz., Dummerstorf 46 (2003), 307-319

BOLLMEIER, S.; MAYER, M.; SIMON, D.:

Entwicklung der genetischen Struktur beim württembergischen Braunvieh. Züchtungskunde 63 (1991), 1-11

BREM, G., BRENIG, B., MÜLLER, M., SPRINGMANN, K., KRÄUSSLICH, H.:

Genetische Vielfalt von Rinderrassen. Verlag Eugen Ulmer, Stuttgart, (1990)

BRODAUF, W.:

Persönliche Mitteilung (2003)

BURROW, H.M.:

The effects of inbreeding in beef cattle. Anim. Breeding Abstr. 61 (1993), 737-751

CASSEL, B.G.; ADAMEC, V.; PEARSON, R.E.:

Effect of incomplete pedigrees on estimates of inbreeding and inbreeding depression for days to first service and summit milk yield in Holsteins and Jerseys. J. Dairy Sci. 86 (2002a), 2967-2976

CASSEL, B.G.; ADAMEC, V.; PEARSON, R.E.:

Maternal and fetal inbreeding depression for 70-day nonreturn and calving rate in Holsteins and Jerseys.

J. Dairy Sci. 86 (2002b), 2977-2983

DGfZ:

Empfehlungen zur Erhaltung lebender Tierbestände einheimischer gefährdeter Nutztierrassen. Züchtungskunde 62 (1992), 77-80

EHLING, C.; SCHMIDT, T.; NIEMANN, H.:

Untersuchungen zur genetischen Struktur und Diversität der Genreserve Deutscher Schwarzbunter Rinder alter Zuchtrichtung. Züchtungskunde 71 (1999), 130-146

ENGELHARDT, I.:

Inzucht, bedeutende Ahnen und Wahrscheinlichkeit für BLAD-Merkmalsträger in der Deutschen Schwarzbuntzucht. Diss. Hannover (1996)

GROENEVELD, E.:

Strategie und Logistik zur verantwortungsvollen Verwaltung der genetischen Diversität in der Nutztierzüchtung. Züchtungskunde 75 (2003), 309-316

HAGGER, C.:

Stand der Inzucht im Schweizer Braunvieh. Landwirtschaft Schweiz 1 (1988), 293-296

KROGMEIER, D.; AUMANN, J.; AVERDUNK, G.:

Untersuchungen zur Inzucht in der Gelbvieh- und Braunviehpopulation in Süddeutschland. Züchtungskunde 69 (1997), 233-243

LUTAAYA, E.; MISZTAL, I.; BERTRAND, J.K.; MABRY, J.W.: Inbreeding in populations with incomplete pedigree. J. Anim. Breed. Genet. 116 (1999), 475-480

MIGLIOR, F.; BURNSIDE, E.B.:

Inbreeding of Canadian Holstein cattle. J. Dairy Sci. 78 (1995), 1163-1167

MIGLIOR, F.; BURNSIDE, E.B.; SULLIVAN, B.P.; SZKOTNICKI, B.; KENNEDY, B.W.:

Trends in inbreeding in Canadian dairy cattle. Proc. $4^{\text {th }}$ World Congress on Genetics applied to Livestock Prod., Edinburgh (1990), 183-186

NAVAJAS, E.A.; URIOSTE, J.I.:

Effects of inbreeding and preweaning growth of Aberdeen-Angus calves. Revista Argentina de Produccion Animal 15 (1995), 880-883

OLLIVIER, L.:

Optimum replacement rates in animal breeding. Anim. Prod. 19 (1974), 257-271 
PIRCHNER, F.:

Schätzung inzuchtwirksamer (effektiver) Populationsgrößen aus Genfrequenzschwankungen bei Bayerischem Fleckvieh und Tiroler Grauvieh. Arch. Tierz., Dummerstorf 45 (2002), 331-339

ROUGHSEDGE, T.; BROTHERSTONE, S.; VISSCHER, P.M.; BOYAZOGLOU, J.; RAFAL, P.; THOMAS, C.; ZJALIC, M.:

Quantifying genetic contributions to a dairy population using pedigree analysis. EAAP Publication 60 (1999), 359-369

SCHMIDT, T.; MAYER, M.; SIMON, D.:

Analyse der westfälischen Rotbuntzucht bezüglich Inzucht, Verwandtschaft und Fremdgenanteil. Züchtungskunde 65 (1993), 102-111

SIMIANER, H.; KÖNIG, S.: Ist Inzucht ein Problem in der deutschen Holsteinzucht? Milchrind H. 3 (2003), 40-43

WIGGANS, G.R.; VAN RADEN, P.M.; ZUURBIER, J.:

Calculation and use of inbreeding coefficients for genetic evaluation of United States dairy cattle. J. Dairy Sci. 78 (1995)), 1584-1590

WREDE, J.; SCHMIDT, T.:

OPTI-MATE. Ein Management-Programm zur Minimierung der Inzucht in gefährdeten Populationen. (2003)

WRIGHT, S.:

Mendelian analysis of the pure breeds of livestock. 1. The measurement of inbreeding and relationship. J. Hered. 14 (1923), 339-348

YOUNG, C.W.; SEYKORA, A.J.:

Estimates of inbreeding and relationship among registered Holstein females in the United States. J. Dairy Sci. 79 (1996), 502-505

Eingegangen: 12.01.2004

Akzeptiert: 27.02.2004

Anschriften der Verfasser

Prof. Dr. GÜNTER BIEDERMANN, Dipl.-Ing. BETTINA OTT,

Dipl.-Ing. KARIN RÜBESAM

Fachgebiet Tierzucht, Universität Kassel

Nordbahnhofstraße 1a

D-37213 Witzenhausen

Dr. FRANZ MAUS

Referat Tierzucht

ALLB Donaueschingen

Moltkestraße 8

D-78166 Donaueschingen 